\title{
Principles of Signal Conversion: A Brief Tutorial
}

\author{
Andri Mirzal \\ Department of Innovation and Technology Management \\ College of Graduate Studies \\ Arabian Gulf University, Kingdom of Bahrain \\ andrim@agu.edu.bh
}

\begin{abstract}
This paper discusses some fundamental aspects in signal conversion for controlling analog plants using digital computer. We especially emphasize on analog to digital (A/D) and digital to analog (D/A) conversion processes. Mathematical models for $A / D$ and $D / A$ conversion processes are derived with the corresponding hardware implementation examples for better understanding about the processes.
\end{abstract}

Keywords: analog to digital, digital to analog, hardware design, signal conversion

\section{Introduction}

Signal conversion is a process for signal conditioning so that it meets the specification requirements in the next processing step [1]. This article discusses about signal conversion for controlling analog plants using digital computer. The discussion emphasizes on analog to digital (A/D) conversion, digital to analog (D/A) conversion, signal sampling, and methods for signal multiplexing.

The General Control Law (GCL) usually is defined using discrete variables in scalar or vector forms [2]. The following equation describes a formula of GCL in difference equation:

$$
e(k T)=\sum_{i=0}^{n} a e((k-i) T)+b e((k-j) T)
$$

where $e(k T)$ denotes discrete signal, $a$ and $b$ are some positive constants, $k$ is a positive integer, and $T$ is sampling interval (see Figure 2 and 3 for an intuitive description of the variables). In practice there are always delays in the conversion process, e.g., delay in analog signal $e(t)$ to discrete signal $e(k T)$ conversion process, delay in arithmetic operation, and delay when converting the discrete signal back to another analog signal [3]. The following summarizes the delays in signal conversion process:

1. Delay in the input (analog to digital conversion): $\tau_{A / D}$

2. Delay in the computation (e.g.: difference equation and error checking computation): $\tau_{C}$

3. Delay in the output (digital to analog conversion): $\tau_{D / A}$

The following formula gives condition that must be met for signal conversion processing in order to guarantee the theoretical assumption about infinitesimal processing time:

$$
\tau_{A / D}+\tau_{C}+\tau_{D / A}<<T
$$

Received (March 3, 2017), Review Result (November 22, 2017), Accepted (November 23, 2017) 


\section{Signal Conversion System}

Signal conversion system is responsible to convert a signal from one form to another form without altering information contained in the signal [1]. This system is only responsible for transforming the physical form of the signal. Based on information preservation capability, signal conversion process can be classified into two classes: lossless conversion and lossy conversion [4]. As the terms implied, in lossless conversion there is no information loss in the converted signal and in lossy conversion there is information loss or reduction in the converted signal. Some examples of lossless conversion are conversion from electrical current to voltage or frequency, electrical voltage to current or frequency, and frequency to electrical voltage and current. And examples of lossy conversion are $\mathrm{A} / \mathrm{D}$ and $\mathrm{D} / \mathrm{A}$ conversion.

\subsection{The A/D Conversion}

The A/D conversion is especially important as the output can be sent to digital computers for further processing and analysis. In some applications, the output of the digital computer (digital signal) can be transformed back to analog signal for controlling analog plant. To improve the quality of conversion, usually antialiasing filtering is applied to analog signal before it is converted to digital signal [5], and smoothing filtering is applied to digital signal before it is converted back to analog signal [6].

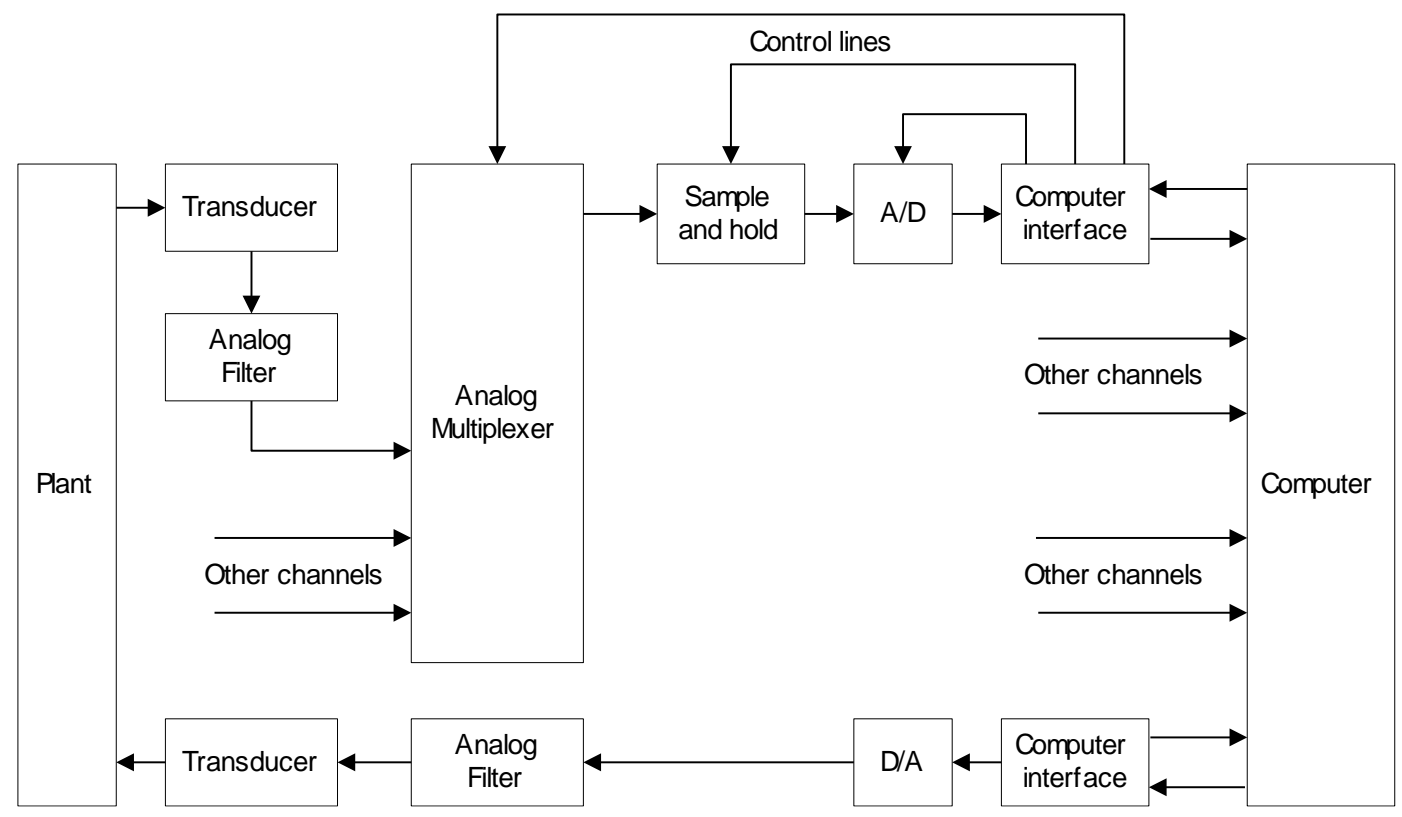

Figure 1. Signal Conversion System

Figure 1 describes a generic signal conversion system where transducer is a device that converts a physical quantity from the analog plant such as pressure, temperature, velocity, light intensity, or position into an electrical signal (current, voltage, or frequency), and vice versa. The input and output ports (serial or parallel) are interfaces that connect the computer with external environment. A multiplexer is a device that is used to manage data traffic between ports [7].

Analog signal from the plant is first read by transducer and then passed to filter for signal conditioning (such as signal amplification and intensification, spectrum conversion, and noise reduction) to meet receiving device specifications. This analog signal is then converted to digital form (binary coded) by digital processor for the next processing stage. After digital manipulation and processing, this signal is transferred to memory and then 
the computer can perform analysis and generate a digital control signal that will be converted to analog control signal for controlling the plant.

Sequencing process in A/D converter is controlled by signals that are generated in the computer interfaces [8]. These control signals can be generated and controlled by either software or hardware (or combination of both). The D/A processing is usually much easier to be controlled because the output is synchronized with the CPU cycle and the data is transferred directly without handshaking process [9].

\subsection{The D/A Conversion}

The signal for controlling plant produced by computer is digital signal in binary form. This digital control signal can be directly used in digital plants such as stepper motor and transistor [10]. If the plant is analog, then the signal must be transformed to analog signal. The digital control signal goes through two conditioning process before it is transformed to analog control signal: coding for determining amplitude of the analog signal for each predetermined interval and holding for generating discrete signal [11]. Figure 2 describes an example of D/A signal conversion. The left picture depicts the signal after the coding process, and the right picture depicts the signal after the holding process (y-axis is the amplitude of the signal and $\mathrm{x}$-axis is the time).
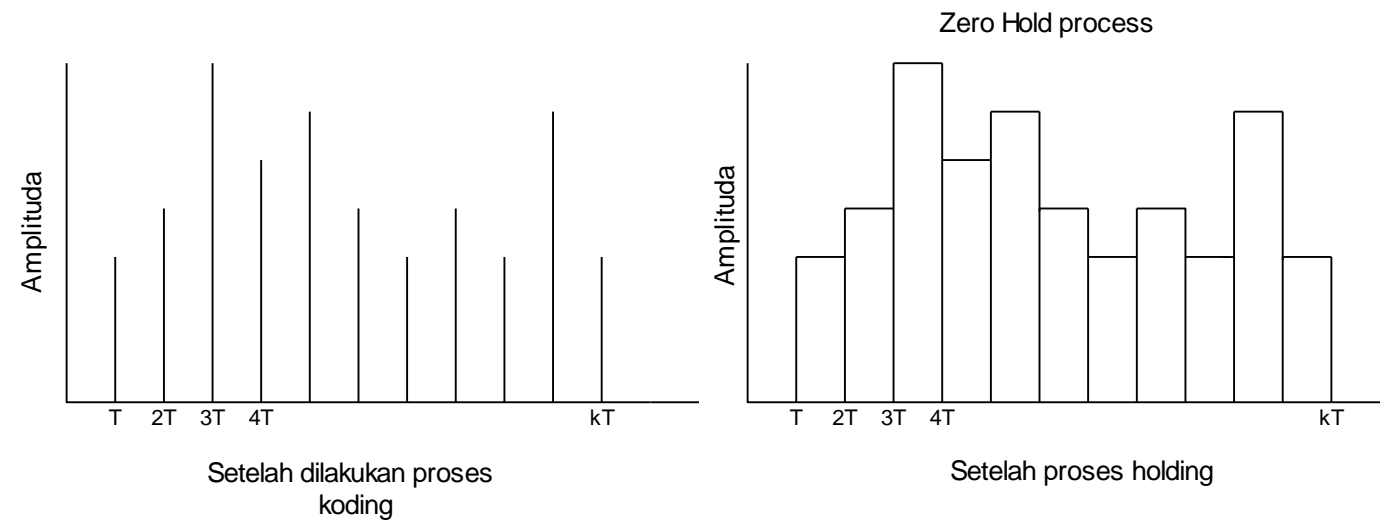

Figure 2. Conditioning Processes in D/A Signal Conversion

The D/A coding process is conducted by reading signal amplitudes information from memory and generating the amplitude for each predetermined interval based on these information (left picture in Figure 2). After all amplitude values are generated for every interval, the signal is held by the hold device to form the discrete signal $g(k T)$ (right picture in Figure 2). Subsequently, the analog signal $g(t)$ is generated from $g(k T)$ by using the following formula:

$$
g(t)=\sum_{k=0}^{M-1} g_{k}\left[u_{-1}(t-k T)-u_{-1}(t-k T-T)\right]
$$

where $u_{-l}$ denotes unit impulse signal. Figure 3 shows the resulting analog signal $g(t)$ from this $\mathrm{D} / \mathrm{A}$ conversion process. 


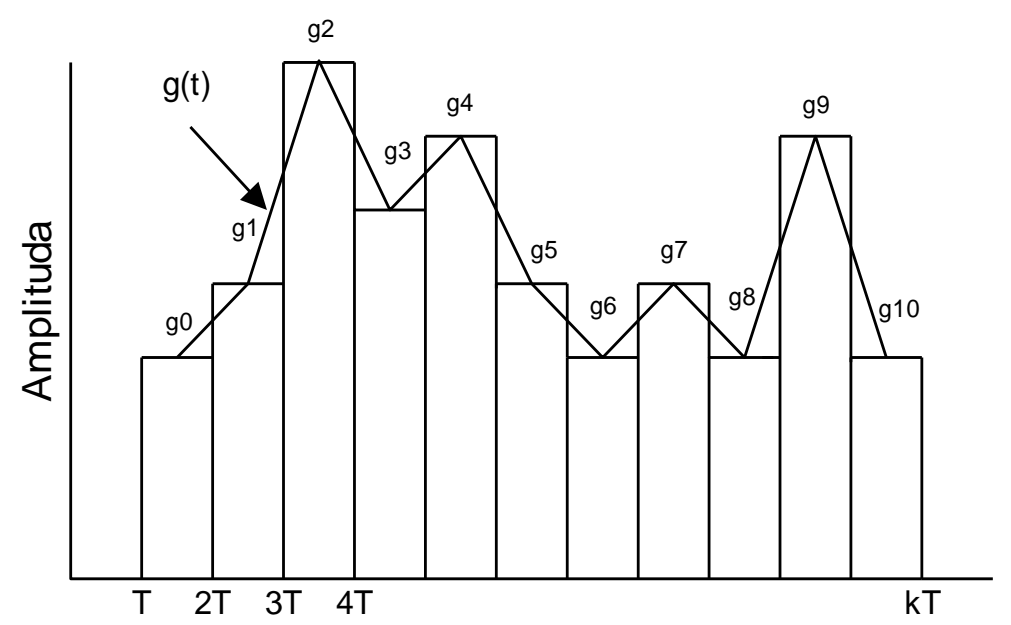

Figure 3. Analog Signal Generation from Discrete Signal

The D/A conversion process described above is performed by D/A converter; a hardware device that performs the conversion process. The output of this device is usually in electrical current or voltage. A time delay $\tau_{D / A}$ is usually added in the D/A converter structure to capture the delay in the conversion process. Figure 4 gives an example of such D/A converter hardware.

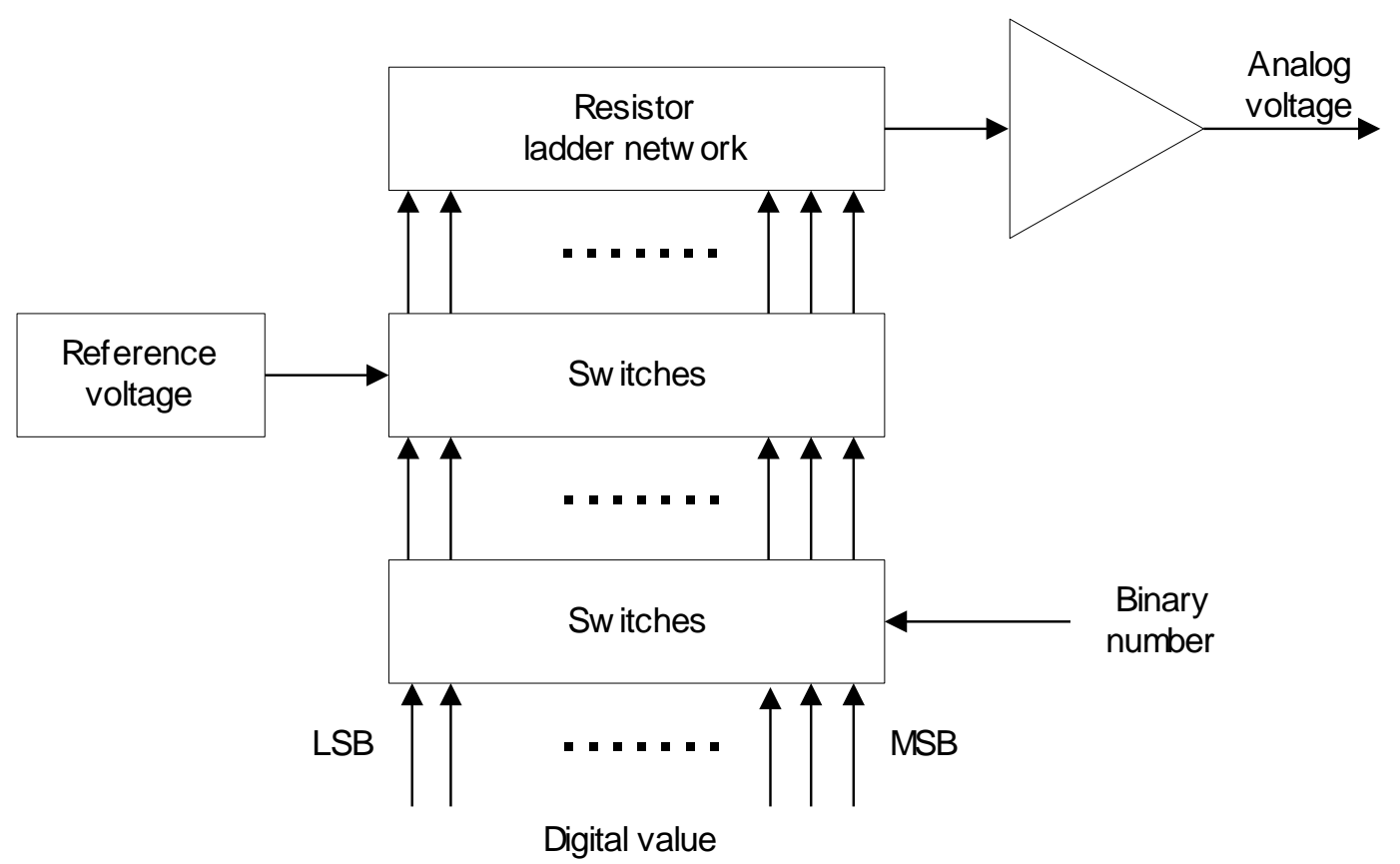

Figure 4. A Hardware Configuration for Performing D/A conversion

As shown in Figure 4, the conversion process from binary signal to analog signal can be physically performed by using register, electronics switches, and passive resistor network. The output voltage $V_{\text {out }}$ in the figure can be represented by:

$$
V_{\text {out }}=V_{\text {ref }}\left(b_{1} 2^{-1}+\cdots+b_{n} 2^{-q}\right)
$$

where $b_{i}$ denotes the value of input in bits from $q$-bit register. Every register bit from most significant bit (MSB) to least significant bit (LSB) is given a weight by the resistor network according to its binary digit. In this case, every $b_{i}$ has a weight of $V_{\text {ref }} / 2^{i}$. 


\section{Input Signal Interfacing}

Transducer or sensor is used to convert a physical quantity from plant into another quantity that can be processed by digital system [12]. The physical quantity to be measured from the plant is usually a continuous analog signal. However, discrete quantities such as the number of event in a time interval e.g. cardiac cycle can also be considered as inputs for transducer.

In this paper, the output from transducer is assumed to be only electrical signals such as current, voltage, or frequency. This output usually needs to be conditioned first to meet the input specification of the receiving $\mathrm{A} / \mathrm{D}$ converter device, e.g., input for ADC 0808/0809 device from Texas Instrument must be voltage in the range of 0-5 V [13]. The signal conditioning process usually involves scaling and shifting operations. In the following we illustrate, with examples, the conditioning process for converting output signal in electrical current, voltage, or frequency from transducer to the corresponding voltage that meets input specification of an $\mathrm{A} / \mathrm{D}$ converter.

\subsection{Current to Voltage Conversion}

For this conversion, the operations that must be performed are current to voltage conversion, scaling, and shifting operations. Mathematically, these three operations can be modeled with the following equation:

$$
V_{\text {out }}=a I_{\text {in }}-b
$$

where $V_{\text {out }}$ denotes output signal from the signal conditioner, $a$ denotes scaling factor, $I_{\text {in }}$ denotes the input, and $b$ denotes shifting factor. The mathematical model above can be implemented by circuit in Figure 5.

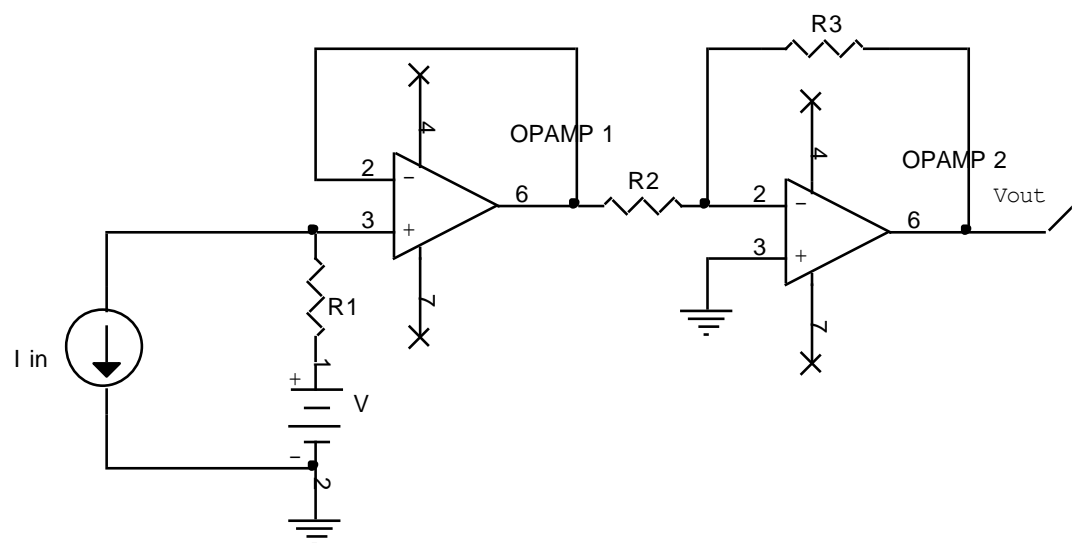

Figure 5. Current to Voltage Converter

The relationship between $V_{\text {out }}$ with $I_{\text {in }}$ in Figure 5 can be described with the following equation.

$$
V_{\text {out }}=-\frac{R_{3}}{R_{2}}\left[V-I_{\text {in }} R_{1}\right]
$$

As shown, the scaling and shifting operations can be performed by adjusting the values of $R_{1}, R_{2}, R_{3}$, and $V$.

\subsection{Voltage to Voltage Conversion}

To condition the output voltage from transducer or sensor to the A/D converter, scaling and shifting operations are necessary. Mathematically, these operations can be modeled with: 


$$
V_{\text {out }}=a V_{\text {in }}-b
$$

This mathematical model can be implemented with the following electrical circuit.

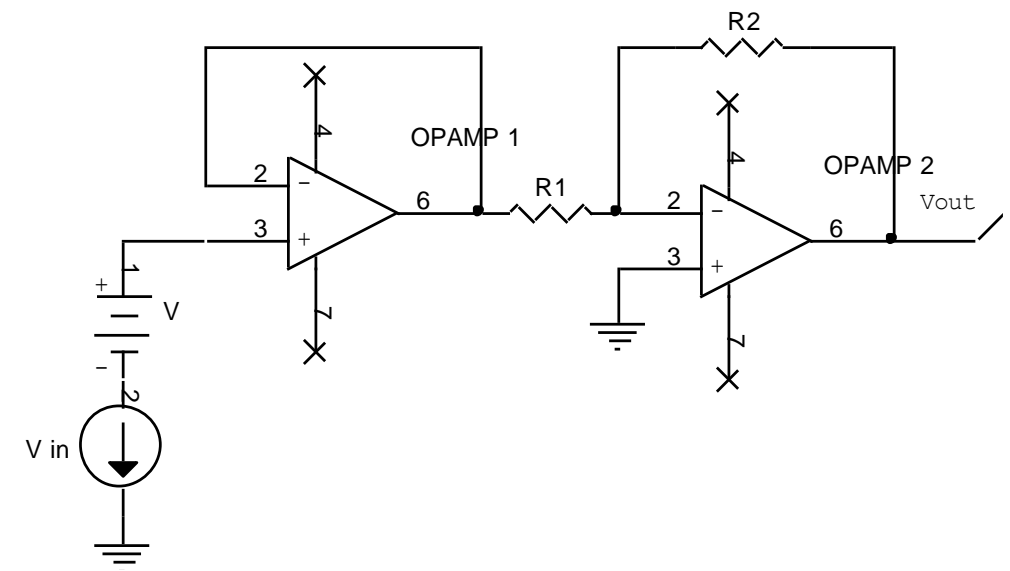

Figure 6. Voltage to Voltage Converter

And the relationship between $V_{\text {out }}$ and $V_{\text {in }}$ can be written with the following equation.

$$
V_{\text {out }}=-\frac{R_{2}}{R_{1}}\left[V-V_{\text {in }}\right]
$$

Accordingly, the scaling and shifting operations can be performed by adjusting the values of $R_{1}, R_{2}$, and $V$.

\subsection{Frequency to Voltage Conversion}

Frequency to voltage conversion is the first operation needs to be performed to condition the signal from transducer or sensor that produces frequency as the output. After this operation, scaling and shifting operations can be applied subsequently to obtain suitable input for the A/D converter. To convert frequency to voltage, LM2907 or LM2917 device from National Semiconductor can be utilized. Figure 7 shows the schematic diagram for LM2907 device.

Based on device specification in the manual [14], the relationship between $V_{\text {in }}$ and $f$ in Figure 7 can be written as:

$$
V_{\text {in }}=V_{c c} R_{1} C_{1} f
$$

$V_{\text {in }}$ is then scaled and shifted so that $\mathrm{A} / \mathrm{D}$ converter can process it further where voltage to voltage converter in Figure 6 can be used to convert $V_{\text {in }}$ to $V_{\text {out }}$. 


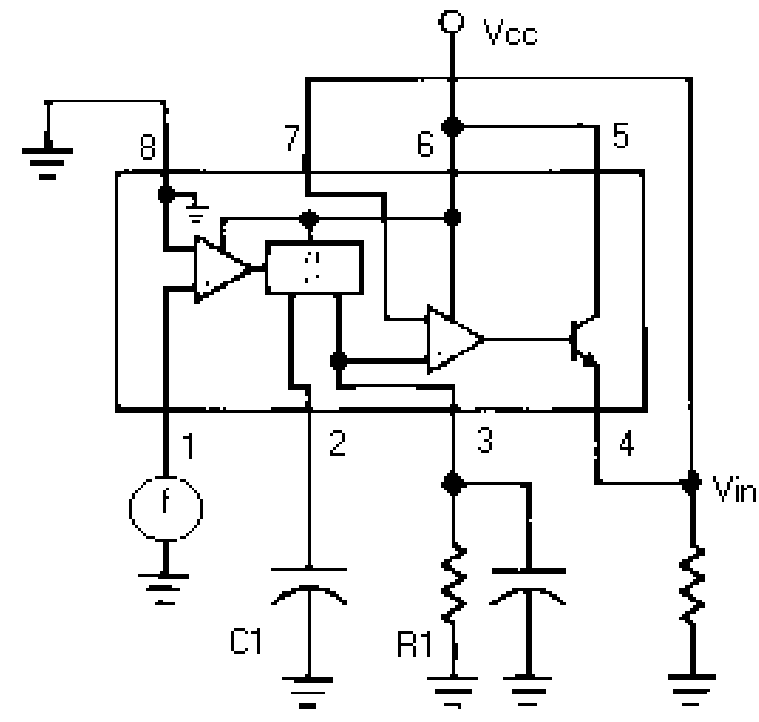

Figure 7. Schematic Diagram of LM2907 [14]

\section{Conclusion}

Signal conversion is a fundamendal process to condition measurement signal or output signal from one device to another device for further processing. The conversion must be done if the measurement or the output signal does not meet input specification of the receving device. The incompatibility is the reason behind signal conversion. As there are many cases where we need to control analog plants with digital computer, understanding $\mathrm{A} / \mathrm{D}$ and $\mathrm{D} / \mathrm{A}$ conversion processes become an indispensable and fundamental knowledge. In this paper, we have discussed some principles in signal conversion. We emphasized the discussion on $\mathrm{A} / \mathrm{D}$ and $\mathrm{D} / \mathrm{A}$ conversion processes and provided mathematical models for the conversion processes with the corresponding hardware implementation for description purpose.

\section{References}

[1] H. Austerlitz, "Data acquisition techniques using PCs", Academic press, (2002).

[2] R. C. Dorf and R. H. Bishop, "Modern control systems", Pearson, (2010).

[3] B. Champagne and F. Labeau, "Discrete Time Signal Processing", Class Notes for the Course ECSE412, Department of Electrical and Computer Engineering, McGill University, (2004).

[4] M. Nelson and J.L. Gailly, "The data compression book (Vol. 2)", New York: M\&t Books, (1996).

[5] C. E. Calosso, C. Clivati and S. Micalizio, "Avoiding Aliasing in Allan Variance: an Application to Fiber Link Data Analysis", IEEE Transactions on Ultrasonics, Ferroelectrics, and Frequency Control, 63(4), (2016), pp. 646-655.

[6] B. Garg and G. K. Sharma, "A quality-aware Energy-scalable Gaussian Smoothing Filter for image processing applications", Microprocessors and Microsystems, 45, (2016), pp. 1-9.

[7] T. Mahmoodi and S. Seetharaman, "Traffic jam: Handling the increasing volume of mobile data traffic", IEEE Vehicular Technology Magazine, vol. 9, no. 3, (2014), pp. 56-62.

[8] L. Bilinskis and K. Sudars, "Digital representation of analog signals by timed sequences of events", Elektronika ir Elektrotechnika, vol. 83, no. 3, (2015), pp. 89-92.

[9] D. Puthal, S. Nepal, R. Ranjan and J. Chen, "A Synchronized Shared Key Generation Method for Maintaining End-to-End Security of Big Data Streams", In Proceedings of the 50th Hawaii International Conference on System Sciences, (2017).

[10] M. Butcher, A. Masi, R. Picatoste and A. Giustiniani, "Hybrid stepper motor electrical model extensions for use in intelligent drives", IEEE Transactions on Industrial Electronics, vol. 61, no.2, (2014), pp. 917 929.

[11] S. Balasubramanian, V. J. Patel and W. Khalil, "Current and emerging trends in the design of digital-toanalog converters", In Design, modeling and testing of data converters, Springer Berlin Heidelberg, (2014), pp. 83-118. 
[12] A. Agarwal and J. Lang, "Foundations of analog and digital electronic circuits", Morgan Kaufmann, (2005).

[13] M. A. Mazidi, J. G. Mazidi and R. D. McKinlay, "The 8051 microcontroller and embedded systems: using Assembly and C", 626, Pearson/Prentice Hall, (2006).

[14] LM2907 and LM2917 Frequency to Voltage Converter. Texas Instruments (retrieved on March 2nd, 2017 from http://www.ti.com/lit/ds/symlink/lm2907-n.pdf).

\begin{abstract}
Author
Andri Mirzal, he received PhD and MSc in Information Science and Technology from Hokkaido University (Japan), and BEng in Electrical Engineering from Institut Teknologi Bandung (Indonesia). His research interests include machine learning, bioinformatics, optimization methods, web search engine, and linear inverse problems. Currently, he is an associate professor in Department of Innovation and Technology Management, College of Graduate Studies, Arabian Gulf University. He was the recipient of Monbukagakusho Scholarship from the Japanese Government for both MSc and PhD programs (2006 - 2011). He also won the National Research Award 2015 in Information and Communication Technology sector from The Research Council of Oman. He has supervised/currently is supervising a number of MSc and PhD students where one of his students was successful in publishing a paper in an IEEE Transactions journal. He was granted several national level research grants by Malaysian government. He has served as a reviewer in some international conferences, research councils such as Research Grant Council of Hong Kong, and journals such as IEEE Transactions on Neural Networks and Learning Systems. He is also an associate editor of the Advances in Engineering Science \& Technology journal (Ology Press).
\end{abstract}

